\title{
Circulating Concentrations of Chemokines in Cord Blood, Neonates, and Adults
}

\author{
SANDRA E. SULLIVAN, SUSAN L. STABA, JASON A. GERSTING, ALAN D. HUTSON, \\ DOUGLAS THERIAQUE, ROBERT D. CHRISTENSEN, DARLENE A. CALHOUN \\ Division of Neonatology [S.E.S., J.A.G.], General Clinical Research Center [A.D.H., D.T.], University of \\ Florida College of Medicine, Gainesville, Florida 32610-0296; Division of Pediatric \\ Hematology/Oncology [S.L.S.], Duke University Medical Center, Durham, North Carolina 27710; \\ Department of Pediatrics [R.D.C.], Division of Neonatology [D.A.C.], University of South Florida, \\ Children's Research Institute, St. Petersburg, Florida 33701
}

\begin{abstract}
ABSTRA
Chemokines are critical for the movement of leukocytes.
Chemotaxis is deficient in neonates, particularly those delivered
prematurely, and this likely contributes to their increased vulner-
ability to sepsis. The concentrations of circulating chemokines in
neonates have not been reported, nor is it known whether low
chemokine concentrations contribute to their defective chemo-
taxis. We hypothesized that serum concentrations of chemokines
1) would be lower in preterm than term neonates, and 2) would
be lower in preterm and term neonates than adults. Samples were
obtained from preterm and term neonates with normal neutrophil
and eosinophil counts, umbilical cord blood samples from preg-
nancies without clinical evidence of intra-amniotic infection, and
healthy adult volunteers. The concentrations of epithelial neutro-
phil activating peptide-78, growth-related oncogene- $\alpha$, eotaxin,
RANTES (regulated upon activation, normal T cell expressed
\end{abstract}
Neonatal sepsis is a life-threatening event affecting 3-5 neonates per 1000 live births (1). The susceptibility of neonates to serious bacterial and fungal infections is related, in part, to quantitative and qualitative neutrophil deficiencies (2). Neutrophil migration, or chemotaxis, involves the recruitment of neutrophils from the circulation, their adherence to vascular endothelial cells, and their migration through the vessel wall to the site of microbial invasion (3). Important elements in this process are the chemotactic cytokines collectively known as chemokines. These are members of a family of homologous proteins with molecular weights in the range of $8-12 \mathrm{kD}(4,5)$.

IL-8 is the most extensively studied chemokine in neonates (6), but no information exists regarding the circulating concentrations of other leukocyte-specific chemokines during the neonatal period. Because preliminary in vitro studies suggest

Received June 22, 2001; accepted November 28, 2001.

Correspondence and reprint requests: Darlene A. Calhoun, D.O., University of South Florida, Children's Research Institute, 140 Seventh Avenue South, CRI Room 2006, St. Petersburg, FL 33701, U.S.A.; e-mail: dcalhoun@hsc.usf.edu

Supported by Grants HD-01180 (D.A.C.), HD-42326 (D.A.C.), and HL-44951 (R.D.C.) from the National Institutes of Health. and secreted), and macrophage inflammatory protein- $1 \alpha$ were measured using specific ELISA. Serum concentrations from preterm infants were either similar to or higher than those measured in term neonates and adults. We conclude that the chemotactic defect observed in premature neonates is not the result of diminished circulating concentrations of any of the specific chemokines we measured. (Pediatr Res 51: 653-657, 2002)
Abbreviations:
ENA-78, epithelial neutrophil activating peptide-78
GRO- $\alpha$, growth-related oncogene- $\alpha$
MIP-1 $\alpha$, macrophage inflammatory protein- $1 \alpha$
RANTES, regulated upon activation, normal $\mathrm{T}$ cell expressed
and secreted

that perturbations in the concentration of chemokines are, in part, responsible for deficiencies in leukocyte chemotaxis (7), we quantified the circulating concentrations of specific leukocyte chemokines in cord blood sera/plasma and in the sera/ plasma of uninfected preterm and term neonates. On the basis of the chemotactic deficiencies of neonates, we hypothesized that the serum concentrations of leukocyte chemokines would 1) be lower in preterm neonates than in term neonates and 2) be lower in term neonates than in adults. Using specific ELISA, we measured the concentrations of neutrophil-specific $\alpha$-chemokines - ENA-78 and GRO- $\alpha$ - as well as eosinophil-, basophil-, and monocyte-specific $\beta$-chemokines-RANTES, eotaxin, and MIP- $1 \alpha$.

\section{MATERIALS AND METHODS}

\section{Subjects}

Preterm $(n=50)$ and term neonates $(n=50)$ without evidence of infection, who had neutrophil and eosinophil counts within normal limits $(8,9)$, were studied during their 
first $3 \mathrm{~d}$ of life. Cord blood sera from preterm $(n=50)$ and term pregnancies $(n=50)$ without clinical evidence of intraamniotic infection and healthy adults were also studied.

\section{Sample Preparation}

ENA-78, GRO- $\alpha$, and RANTES. Samples of serum or plasma (citrate, EDTA, or heparin as the anticoagulant) were collected after centrifuging blood samples for $10 \mathrm{~min}$ at 1000 $\times g$. Serum was immediately separated into aliquots and stored at $-20^{\circ} \mathrm{C}$ until assay. When plasma was used, an additional centrifugation step was performed at $10,000 \times g$ for $10 \mathrm{~min}$ to insure removal of platelets. All samples for ENA-78 were then diluted at least 2-fold using Calibrator Diluent RD6-1 (R \& D Systems, Minneapolis, MN, U.S.A.). All samples for RANTES were diluted at least 50-fold using Calibrator Diluent RD5H (R \& D Systems).

Eotaxin and MIP-1 $\alpha$. Samples of serum or plasma (citrate or EDTA as the anticoagulant) were collected after centrifuging for $10 \mathrm{~min}$ at $1000 \times \mathrm{g}$. The samples were then separated into aliquots and stored at $-20^{\circ} \mathrm{C}$ until assay.

All samples were thawed on ice at the time of assay. No repeated freezing and thawing of samples occurred. Informed consent was obtained from all women who donated cord blood and from all adult volunteers. In regard to the samples from term and preterm neonates, the only information obtained about the infants was gestational age and postnatal age; no information was collected about the clinical characteristics. All specimens from neonates were the residual blood from samples collected for laboratory tests, after completion of these tests. Therefore, informed consent did not need to be obtained according to the discarded specimen policy at the University of Florida.

\section{ELISA Determinations}

Serum concentrations of each chemokine were quantified by specific ELISA using a double-antibody sandwich technique, following the manufacturer's instructions (Quantikine ENA78 , GRO- $\alpha$, RANTES, eoxtaxin, and MIP- $1 \alpha, \mathrm{R} \& \mathrm{D}$ Systems). The volume of sample required and the upper and lower limits of detection are shown in Table 1.

\section{Data Analysis}

Descriptive statistics are given by the median and quartiles. Overall group comparisons between preterm neonates, term neonates, preterm cord blood, term cord blood, and adults were carried out for each chemokine using the Kruskal-Wallis rank-

Table 1. Relevant information for each chemokine ELISA

\begin{tabular}{lccc}
\hline Chemokine & Volume $(\mu \mathrm{L})$ of serum required & $\begin{array}{c}\text { Lower limit } \\
\text { of detection } \\
(\mathrm{pg} / \mathrm{mL})\end{array}$ & $\begin{array}{c}\text { Upper limit } \\
\text { of detection } \\
(\mathrm{pg} / \mathrm{mL})\end{array}$ \\
\hline ENA-78 & 50 (requires 2-fold dilution) & 62.4 & 4000 \\
GRO- $\alpha$ & 200 & 31.2 & 1000 \\
Eotaxin & 50 & 15.6 & 1000 \\
MIP-1 $\alpha$ & 200 & 46.9 & 1500 \\
RANTES & 100 (requires 50-fold dilution) & 156 & 100000 \\
\hline
\end{tabular}

based tests. If the overall $p$ value was $\leq 0.05$, pairwise comparisons were carried out using the Bonferroni correction. Values above the upper limit of detection were truncated to the limit of detection for the rank analysis.

\section{RESULTS}

The concentrations of each chemokine are shown in Figure 1. Box plots, a five-number summary including the minimum, the lower quartile (25th percentile), median, upper quartile (75th percentile), and maximum values, were used to display the data.

ENA-78. Preterm cord blood and term cord blood concentrations of ENA-78 were higher than preterm neonatal and adult concentrations $(p<0.005$ for each). There was no difference when preterm concentrations were compared with term $(p=0.37)$, or when either preterm or term concentrations were compared with adult values $(p=0.72$ and 0.58 , respectively).

GRO- $\boldsymbol{\alpha}$. Preterm cord blood concentrations were higher than concentrations in adults $(p<0.005)$ but similar to concentrations in term cord blood. There was no difference between preterm cord blood concentrations and preterm neonatal concentrations $(p=0.08)$. Whereas GRO- $\alpha$ concentrations in preterm neonates were higher than adult concentrations $(p<$ $0.005)$, there was no difference between preterm and term neonatal concentrations $(p=0.18)$ or between term neonatal concentrations and adult concentrations $(p=0.08)$.

RANTES. Concentrations of RANTES in preterm cord blood were lower than those in term cord blood $(p<0.005)$. Preterm and term neonatal concentrations were similar to each other $(p=0.06)$ and similar to adult RANTES concentrations ( $p=0.40$ and 0.12 , respectively).

Eotaxin. Concentrations of eotaxin in preterm cord blood, term cord blood, and term neonates were lower than concentrations in adults ( $p<0.005$ for each). However, there were no significant differences between eotaxin concentrations in preterm neonates when compared with term neonates $(p=0.16)$ or adults $(p=0.26)$.

MIP-1 $\alpha$. All cord blood and neonatal concentrations of MIP- $1 \alpha$ were higher than concentrations in adults $(p<0.005)$. Whereas term neonatal concentrations were higher than term cord blood MIP- $1 \alpha$ concentrations $(p<0.005)$, preterm neonatal and preterm cord blood concentrations were similar $(p=$ $0.25)$. Preterm neonatal concentrations of MIP- $1 \alpha$ were similar to term neonatal concentrations $(p=0.03)$, and preterm cord blood concentrations were similar to term cord blood concentrations $(p=0.01)$.

\section{DISCUSSION}

Each of the leukocyte chemokines we studied was measurable in the serum or plasma of preterm and term neonates. Concentrations of these $\alpha$-chemokines in preterm neonates were either higher than or the same as those in term neonates or adults. Therefore, the chemotactic defect of neonatal neutrophils is not the result of a deficiency in the circulating concentrations of the $\alpha$-chemokines we evaluated. Because ELISA for granulocyte chemotactic peptide-2, neutrophil acti- 

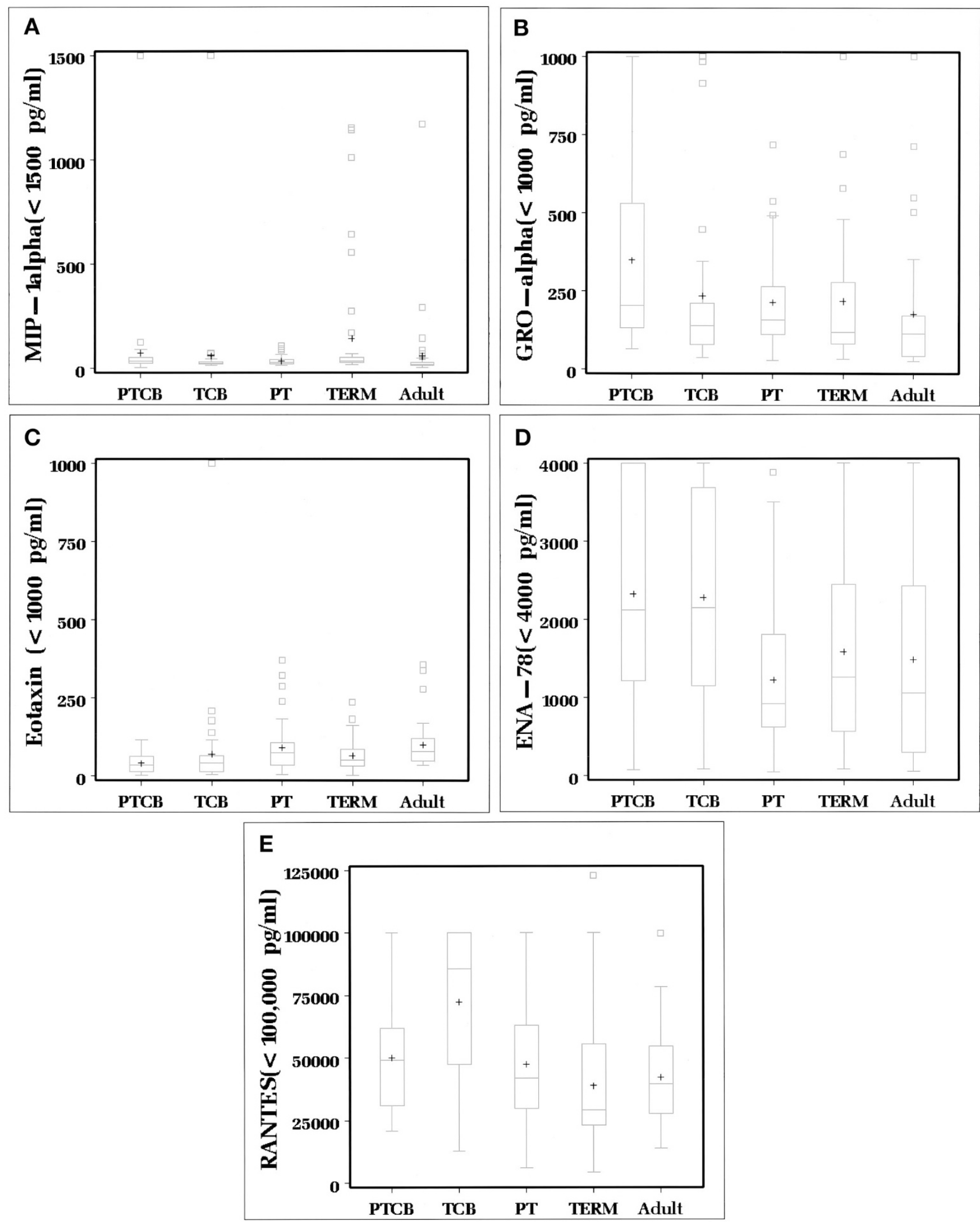

Figure 1. Concentrations of MIP- $1 \alpha$, GRO- $\alpha$, eotaxin, ENA-78, and RANTES in preterm cord blood (PTCB), term cord blood (TCB), preterm serum/plasma $(P T)$, term serum/plasma $($ TERM $)$, and adult serum/plasma (Adult) presented as box plots. The means are marked by asterisks $(*)$, and the medians are designated by horizontal lines through the central box. The upper and lower hinges $(H)$ comprise the edges of the central box representing the interquartile range. The $\mathrm{H}$ spread is the absolute value of the differences between the values of the two hinges. The fences $(\perp)$ show the range of the values that fall within $1.5 \mathrm{H}$ spreads of the hinges. Values outside the inner fences $( \pm 1.5 \mathrm{H}$ spread) are plotted as open boxes $(\square)$. 
vating peptide-2, and lipopolysaccharide (LPS)-induced CXC (cysteine-X-cysteine) chemokine were not commercially available at the time of this study, we cannot rule out that deficiencies in other $\alpha$-chemokines (4) might be responsible for the chemotactic defect of neonatal neutrophils. Another potential explanation for $\alpha$-chemokine concentrations in preterm infants not differing from those in term infants and adults could be related to the process of preterm delivery. Cytokine concentrations in umbilical cord blood can be higher than in maternal blood (10).

Concentrations of the $\beta$-chemokines, RANTES and eotaxin (specific for eosinophils, basophils, and monocytes), were noted to be less in certain comparisons. RANTES concentrations in preterm cord blood were significantly less than concentrations in term cord blood, and eotaxin concentrations in preterm and term cord blood and term neonatal samples were all significantly less than adult concentrations.

Little is known regarding what directs the migration of leukocytes under unperturbed conditions, during stress, or during infection. Even less is known about circulating concentrations, or production, of chemokines during the neonatal period. Dammann et al. (11) measured MIP-1 $\alpha$ and RANTES along with 14 other markers of inflammation in cord blood and postnatal serum from extremely low gestational age newborn infants. Using recycling immunoaffinity chromatography, they found that MIP- $1 \alpha$ and RANTES were considerably higher in preterm samples than published values in term newborns $(8.2$ and $11.4 \mathrm{pg} / \mathrm{mL}$ versus 5.0 and $5.0 \mathrm{pg} / \mathrm{mL}$, for preterm and term MIP- $1 \alpha$ and RANTES, respectively) (12). These results differ from our results in that we found no difference between preterm and term neonatal MIP- $1 \alpha$ concentrations or RANTES concentrations. Several potential explanations exist. First, these differences might reflect differences in the assays used to determine chemokine concentrations in each situation. Recycling immunoaffinity chromatography utilizes whole blood samples, whereas the ELISA in this study used serum or plasma samples. Second, Dammann and colleagues also chose a more select group of preterm neonates ( $<28 \mathrm{wk}$ ), whereas our study population of preterm neonates included all neonates $<37$ wk. Finally, we excluded any samples from women with known intra-amniotic infection. The influence of these discrete variables merits further investigation.

The production of RANTES and MIP- $1 \alpha$ in vitro by cord blood mononuclear cells was examined by Hariharan et al. (7). They found a decreased ability of mononuclear cells in term cord blood to secrete RANTES when stimulated with LPS when compared with adult mononuclear cells. RANTES is predominantly produced by a subset of lymphocytes $\left(\mathrm{CD}^{+} /\right.$ $\mathrm{CD} 45 \mathrm{RO}^{+}$) that are fewer in term cord blood than in adult blood. Hariharan and colleagues hypothesized that the decreased number of these cells might explain the poor secretion of RANTES in term cord blood samples. In addition, they did not observe a difference in the production of MIP- $1 \alpha$ when LPS-stimulated mononuclear cells from term cord blood samples were compared with adult samples. The findings by Hariharan and colleagues appear inconsistent with our reported circulating concentrations of RANTES and MIP- $1 \alpha$. One possible explanation for this is that we measured concentrations of chemokines in noninfected subjects, and they measured the production of chemokines in LPS-stimulated cells.

Another potential explanation for the differences between our study and that of Hariharan et al. (7) is that, as with other cytokines, in vitro stimulation of mononuclear cells as a measure of the ability of cells to produce cytokines might not correlate with in vivo cytokine production. Schibler et al. (13) found that in vitro production of granulocyte colonystimulating factor by monocytes stimulated with LPS was lower using cells from preterm neonates compared with term neonates and adults. They speculated that this defect in production of granulocyte colony-stimulating factor might contribute to a defective up-regulation of neutrophil production and function during infection. However, when granulocyte colony-stimulating factor concentrations were measured in the serum of neonates with neutropenia and confirmed bacterial sepsis, they were significantly higher than those of neonates who were neutropenic and uninfected (14).

Studies of circulating concentrations of chemokines in adults during sepsis suggest that perturbations in chemokine concentrations occur. Cummings et al. (15) evaluated the plasma concentrations of IL-8, GRO- $\alpha$, and ENA-78 in adults during sepsis. The median IL-8 concentration in patients with sepsis was $157 \mathrm{pg} / \mathrm{mL}$, whereas levels were undetectable in noninfected subjects $(p<0.005)$. The median concentration of GRO- $\alpha$ was $170 \mathrm{pg} / \mathrm{mL}$ in the plasma of patients with sepsis compared with $30 \mathrm{pg} / \mathrm{mL}$ in controls $(p=0.07)$. In contrast, the median concentration of ENA-78 did not differ in patients with and without sepsis. Knapp et al. (16) evaluated the prognostic value of MIP- $1 \alpha$, transforming growth factor- $\beta 2$, soluble endothelial leukocyte adhesion molecule-1, and soluble vascular adhesion molecule-1 in patients with Gram-positive sepsis. Concentrations of MIP- $1 \alpha$ were significantly higher than concentrations in healthy control patients ( $>50 \mathrm{pg} / \mathrm{mL}$ versus $25 \mathrm{pg} / \mathrm{mL}$ ). Much remains to be learned about the expression of chemokines in adults and neonates during infection.

Chemotaxis is a complicated process involving activated cells, intact functional receptors, and corresponding ligands. The influence of circulating chemokines on leukocyte chemotaxis is only one element of this process. The present study demonstrates that chemokines are measurable in cord blood serum and neonatal serum and that concentrations are generally similar to those found in adults.

\section{REFERENCES}

1. Kaushik SL, Parmar VR, Grover N, Grover PS, Kaushik R 1998 Neonatal sepsis in hospital born babies. J Commun Dis 30:147-152

2. Carr R 2000 Neutrophil production and function in newborn infants. Br J Haematol 110:18-28

3. Maghazachi AA 2000 Intracellular signaling events at the leading edge of migrating cells. Int J Biochem Cell Biol 32:931-943

4. Luster AD 1998 Mechanisms of disease: chemokines-chemotactic cytokines that mediate inflammation. N Engl J Med 338:436-445

5. Youn BS, Mantel C, Broxmeyer HE 2000 Chemokines, chemokine receptors and hematopoiesis. Immunol Rev 177:150-174

6. Berner R, Niemeyer CM, Leititis JU, Funke A, Schwab C, Rau U, Richter K, Tawfeek MS, Clad A, Brandis M 1998 Plasma levels and gene expression of granulocyte colony-stimulating factor, tumor necrosis factor-alpha, interleukin (IL)-1beta, IL-6, IL- 8, and soluble intercellular adhesion molecule-1 in neonatal early onset sepsis. Pediatr Res 44:469-477

7. Hariharan D, Ho W, Cutilli J, Campbell DE, Douglas SD 2000 C-C chemokine profile of cord blood mononuclear cells: selective defect in RANTES production. Blood 95:715-718 
8. Weinberg AG, Rosenfeld CR, Manroe BL, Browne R 1985 Neonatal blood cell count in health and disease. II. Values for lymphocytes, monocytes, and eosinophils. J Pediatr 106:462-466

9. Mouzinho A, Rosenfeld CR, Sanchez PJ, Risser R 1994 Revised reference ranges for circulating neutrophils in very-low-birth-weight neonates. Pediatrics $94: 76-82$

10. Salafia CM, Sherer DM, Spong CY, Lencki S, Eglinton GS, Parkash V, Marley E, Lage JM 1997 Fetal but not maternal serum cytokine levels correlate with histologic acute placental inflammation. Am J Perinatol 14:419-422

11. Dammann O, Phillips TM, Allred En, O'Shea M, Paneth N, Van Marter LJ, Bose C, Ehrenkranz RA, Bednarek FJ, Naples M, Leviton A 2000 Mediators of fetal inflammation in extremely low gestational age newborns. Cytokine 13:234-239

12. Nelson KB, Dambrosia JM, Grether JK, Phillips TM 1998 Neonatal cytokines and coagulation factors in children with cerebral palsy. Ann Neurol 44:665-675
13. Schibler KR, Liechty KW, White WL, Christensen RD 1993 Production of granulocyte colony-stimulating factor in vitro by monocytes from preterm and term neonates. Blood 82:2478-2484

14. Calhoun DA, Lunoe M, Du Y, Hutson AD, Veerman M, Christensen RD 2000 Granulocyte colony-stimulating factor serum and urine concentrations in neutropenic neonates before and after intravenous administration of recombinant granulocyte colony-stimulating factor. Pediatrics 105:392-397

15. Cummings CJ, Martin TR, Frevert CW, Quan JM, Wong VA, Mongovin SM, Hagen TR, Steinberg KP, Goodman RB 1999 Expression and function of the chemokine receptors CXCR1 and CXCR2 in sepsis. J Immunol 162:2341-2346

16. Knapp S, Thalhammer F, Locker GJ, Laczika K, Hollenstein U, Frass M, Winkler S, Stoiser B, Wilfing A, Burgmann H 1998 Prognostic value of MIP-1 alpha, TGF-beta 2, sELAM-1, and sVCAM-1 in patients with Gram-positive sepsis. Clin Immunol Immunopathol 87:139-144 